\title{
CHARACTERIZATION OF A CLASS OF INFINITE MATRICES WITH APPLICATIONS
}

\author{
P.N. NatARajan
}

In this paper, $K$ denotes a complete, non-trivially valued, nonarchimedean field. The class $\left(z_{\alpha}, z_{\alpha}\right)$ of infininite matrices transforming sequences over $K$ in $\tau_{\alpha}$ to sequences in $l_{\alpha}$ is characterized. Further a Mercerian theorem is proved in the context of the Banach algebra $\left(z_{\alpha}, z_{\alpha}\right), \alpha \geq 1$ and finally a steinhaus type result is proved for the space $\tau_{\alpha}$. In the case of $\mathbb{R}$ or $C$, on the other hand, the best known result so far seems to be a characterization of positive matrix transformations of the class $\left(z_{\alpha}, z_{\beta}\right)$, $\infty>\alpha \geq \beta>1$.

\section{Introduction.}

$K$ denotes a complete, non-trivially valued field, that is $K=\mathbb{R}$ (the field of real numbers) or $\phi$ (the field of complex numbers) or a complete, non-trivially valued, non-archimedean field.

If $X, Y$ are sequence spaces with elements whose entries are in $K$ and if $A=\left(a_{n k}\right), a_{n k} \in K, n, k=0,1,2, \ldots$ is an infinite matrix, we

Received 16 December 1985.

Copyright Clearance Centre, Inc. Serial-fee code: 00049727/86 $\$ A 2.00+0.00$. 
write $A \in(X, Y)$ if $(A x)_{n}=\sum_{k=0}^{\infty} a_{n k} x_{k}$ is defined, $n=0,1,2, \ldots$ and the sequence $A x=\left\{(A x)_{n}\right\} \in Y$, for every $x=\left\{x_{k}\right\} \in X . A x$ is called the $A$ transform of $x$.

The main result of this paper is the characterization of infinite matrices belonging to $\left(z_{\alpha}, z_{\alpha}\right), \alpha>0$, where

$$
z_{\alpha}=\left\{x=\left\{x_{k}\right\}, x_{k} \in K, k=0,1,2, \ldots, \sum_{k=0}^{\infty}\left|x_{k}\right|^{\alpha}<\infty\right\},
$$

where $K$ is a complete, non-trivially valued, non-archimedean field. Because of the fact that there is, as such, no classical analogue for this result, this result is interesting. When $K=\mathbb{R}$ or $\mathbb{}$, a complete characterization of the class $\left(z_{\alpha}, z_{\beta}\right)$ of infinite matrices, $\alpha, \beta \geq 2$, does not seem to be available in the literature. Even a recent result [5] in this direction characterizes only non-negative matrices in $\left(z_{\alpha}, \tau_{\beta}\right)$, $\alpha \geq \beta>1$. When $K=\mathbb{R}$ or $C$, a known simple sufficient condition ([6], p.174, Theorem 9) for an infinite matrix $A$ to belong to $\left(l_{\alpha}, z_{\alpha}\right)$ is

$$
A \in\left(z_{\infty}, z_{\infty}\right) \cap\left(z_{1}, z_{1}\right)
$$

Sufficient conditions or necessary conditions for $A \in\left(\tau_{\alpha}, Z_{\beta}\right)$ when $K=\mathbb{R}$ or $\Phi$ are available in literature (see for example [11]). Necessary and sufficient conditions for $A \in\left(z_{1}, z_{1}\right)$ are due to Mears [7] (for alternate proofs, see Knopp and Lorentz [4], Fridy [2]).

From the characterization mentioned at the outset, it is then deduced that the Cauchy product of two sequences in $Z_{\alpha}, \alpha>0$, is again in $l_{\alpha}$. This result fails to hold for $\alpha>1$ when the field is $\mathbb{R}$ or $\mathbb{W}$. In Section 3 we obtain a Mercerian theorem by considering the structure of the space $\left(z_{\alpha}, z_{\alpha}\right), \alpha \geq 1$, of matrices. In section 4 we study certain Steinhaus type theorems involving the space $\tau_{\alpha}$.

\section{Characterization of matrices in $\left(z_{\alpha}, z_{\alpha}\right), \alpha>0$.}

THEOREM 2.1. If $A=\left(a_{n k}\right), a_{n k} \in K, n, k=0,1,2, \ldots$ where $K$ is a complete, non-trivially valued, non-archimedecon field, 
$A \in\left(z_{\alpha}, z_{\alpha}\right), \alpha>0$ if and only if

$$
\sup _{k \geq 0} \sum_{n=0}^{\infty}\left|a_{n k}\right|^{\alpha}<\infty \text {. }
$$

Proof. Since $|$.$| is a non-archimedean valuation, we first observe$ that

$$
\left.|| a\right|^{\alpha}-|b|^{\alpha}|\leq| a+\left.b\right|^{\alpha} \leq|a|^{\alpha}+|b|^{\alpha}, \alpha>0 .
$$

(Sufficiency). If $x=\left\{x_{k}\right\} \in Z_{\alpha}, \sum_{k=0}^{\infty} a_{n k} x_{k}$ converges, $n=0,1,2, \ldots$, since $x_{k} \rightarrow 0, k \rightarrow \infty$ and $\sup _{n, k}\left|a_{n k}\right|<\infty$ by (2.1). Also,

$$
\begin{aligned}
\sum_{n=0}^{\infty}\left|(A x)_{n}\right|^{\alpha} & \leq \sum_{n=0}^{\infty} \sum_{k=0}^{\infty}\left|a_{n k}\right|^{\alpha}\left|x_{k}\right|^{\alpha} \\
& \leq\left(\sum_{k=0}^{\infty}\left|x_{k}\right|^{\alpha}\right)\left(\sup _{k \geq 0} \sum_{n=0}^{\infty}\left|a_{n k}\right|^{\alpha}\right) \\
& <\infty,
\end{aligned}
$$

so that $\left\{(A x)_{n}\right\} \in Z_{\alpha}$.

(Necessity). Suppose $A \in\left(z_{a}, z_{a}\right)$. We first note that $\sup _{k \geq 0}\left|a_{n k}\right|^{\alpha}=B_{n}<\infty, n=0,1,2, \ldots$. For, if for some $m$, $\sup _{k \geq 0}\left|a_{m k}\right|^{\alpha}=\infty$, then, we can choose a strictly increasing sequence $\{k(i)\}$ of positive integers such that $\left|a_{m, k(i)}\right|^{\alpha}>i^{2}, i=1,2, \ldots$. If the sequence $\left\{x_{k}\right\}$ is defined by

$$
\begin{aligned}
x_{k} & =\frac{1}{a_{m, k(i)}}, k=k(i) \\
& =0, k \neq k(i)
\end{aligned}\left\{\begin{array}{l}
, i=1,2, \ldots,
\end{array}\right.
$$$$
\left\{x_{k}\right\} \in \tau_{\alpha} \text {, for }, \sum_{k=0}^{\infty}\left|x_{k}\right|^{\alpha}=\sum_{i=1}^{\infty}\left|x_{k(i)}\right|^{\alpha}<\sum_{i=1}^{\infty} \frac{1}{i^{2}}<\infty \text {, }
$$

while $a_{m, k(i)} x_{k(i)}=1 \nrightarrow 0, i \longrightarrow \infty$ which is contradiction. 
Since $(A x)_{n}=a_{n k}$ for the sequence $x=\left\{x_{n}\right\}, x_{n}=0, n \neq k, x_{k}=1$ and $\left\{(A x)_{n}\right\} \in Z_{\alpha}$,

$$
\mu_{k}=\sum_{n=0}^{\infty}\left|a_{n k}\right|^{\alpha}<\infty, k=0,1,2, \ldots .
$$

Suppose $\left\{\mu_{k}\right\}$ is unbounded. Choose a positive integer $k(1)$ such that

$$
\mu_{k(1)}>3 \text {. }
$$

Then choose a positive integer $n(1)$ such that

$$
\sum_{n=n(1)+1}^{\infty}\left|a_{n, k(1)}\right|^{\alpha}<1,
$$

so that

$$
\sum_{n=0}^{n(1)}\left|a_{n, k(1)}\right|^{\alpha}>2 .
$$

More generally, given the positive integers $k(j), n(j), j \leq m-1$, choose positive integers $k(m), n(m)$ such that $k(m)>k(m-1), n(m)>$ $n(m-1)$,

$$
\begin{gathered}
\sum_{n=n(m-2)+1}^{n(m-1)} \stackrel{\sum}{k=k(m)}_{n}^{\infty} B_{n} k^{-2}<1, \\
\mu_{k(m)}>2 \sum_{n=0}^{n(m-1)} B_{n}+\rho^{-\alpha} m^{2}\left\{2+\sum_{i=1}^{m-1} i^{-2} \mu_{k(i)}\right\}
\end{gathered}
$$

and

$$
\sum_{n=n(m)+1}^{\infty}\left|a_{n, k(m)}\right|^{\alpha}<\sum_{n=0}^{n(m-1)} B_{n},
$$

where, since $K$ is non-trivially valued, there exists $\pi \in K$ such that $0<\rho=|\pi|<1$.

$$
\begin{aligned}
& \underset{n=n(m-1)+1}{n(m)}\left|a_{n, k(m)}\right|^{\alpha}=\mu_{k(m)}-\sum_{n=0}^{n(m-1)}\left|a_{n, k(m)}\right|^{\alpha}-\sum_{n=n(m)+1}^{\infty}\left|a_{n, k(m)}\right|^{\alpha} \\
& >2 \sum_{n=0}^{n(m-1)} B_{n}+\rho^{-\alpha} m^{2}\left\{2+\sum_{i=1}^{m-1} i^{-2} \mu_{k(i)}\right\}-\sum_{n=0}^{n(m-1)} B_{n}-\sum_{n=0}^{n(m-1)} B_{n} \\
& =\rho^{-\alpha} m^{2}\left\{2+\sum_{i=1}^{m-1} i^{-2} \mu_{k(i)}\right\} \text {. }
\end{aligned}
$$


For every $i=1,2, \ldots$, there exists a non-negative integer $\lambda(i)$ such that

$$
\rho^{\lambda(i)+1} \leq i^{-2 / \alpha}<\rho^{\lambda(i)} .
$$

Define the sequence $x=\left\{x_{k}\right\}$ as follows.

$$
\begin{aligned}
& x_{k}=\pi^{\lambda(i)+1}, k=k(i), k \neq k(i), i=1,2, \ldots . \\
&\left.=0, x_{k}\right\} \in l_{\alpha}, \text { for }, \sum_{k=0}^{\infty}\left|x_{k}\right|^{\alpha}=\sum_{i=1}^{\infty}\left|x_{k(i)}\right|^{\alpha} \leq \sum_{i=1}^{\infty} \frac{1}{i^{2}}<\infty .
\end{aligned}
$$

However, using (2.2),

$$
\sum_{n=n(m-1)+1}^{n(m)}\left|(A x){ }_{n}\right|^{\alpha} \geq \Sigma_{1}-\Sigma_{2}-\Sigma_{3} .
$$

where

$$
\begin{aligned}
& \Sigma_{1}=\sum_{n=n(m-1)+1}^{n(m)}\left|a_{n, k(m)}\right|^{\alpha}\left|x_{k(m)}\right|^{\alpha}, \\
& \Sigma_{2}=\sum_{n=n(m-1)+1}^{n(m)} \sum_{i=1}^{m-1}\left|a_{n, k(i)}\right|^{\alpha}\left|x_{k(i)}\right|^{\alpha}, \\
& \Sigma_{3}=\underset{n=n(m-1)+1}{n} \sum_{i=n+1}^{\infty}\left|a_{n, k(i)}\right|^{\alpha}\left|x_{k(i)}\right|^{\alpha} .
\end{aligned}
$$

Now

$$
\begin{aligned}
\Sigma_{1} & =\sum_{n=n(m-1)+1}^{n(m)}\left|a_{n, k(m)}\right|^{\alpha} \rho(\lambda(m)+1) \alpha \\
& \geq \rho^{\alpha} \sum_{n=n(m-1)+1}^{n(m)}\left|a_{n, k(m)}\right|^{\alpha} m^{-2} \\
& =2+\sum_{i=1}^{m-1} i_{k(i)}^{-2} \mu_{k}, \\
\Sigma_{2} & =\sum_{n=n(m-1)+1} \sum_{i=1}^{\sum_{n=1}}\left|a_{n, k(i)}\right|^{\alpha} \rho^{(\lambda(i)+1) \alpha} \\
& \leq \sum_{i=1}^{m-1} \sum_{i=n(m-1)}^{n} \sum_{n=1}\left|a_{n, k(i)}\right|^{\alpha}
\end{aligned}
$$




$$
\begin{aligned}
& \leq \sum_{i=1}^{m-1} i^{-2} \mu_{k(i)}, \\
\Sigma_{3} & \sum_{n=n(m-1)+1}^{n(m)} \sum_{i=m+1}^{\infty}\left|a_{n, k(i)}\right|^{\alpha} \rho(\lambda(i)+1) \alpha \\
& \leq \sum_{n=n(m-1)+1}^{n(m)} \sum_{i=k(m+1)}^{\infty} B_{n} i^{-2}
\end{aligned}
$$

$$
<1 \text {. }
$$

From (2.3) to (2.5), we have,

$$
\sum_{n=n(m-1)+1}^{n(m)}\left|\left(A x_{n}\right)\right|^{\alpha}>1, m=2,3, \ldots .
$$

This shows that $\left\{(A x)_{n}\right\} \notin Z_{\alpha}$ while $\left\{x_{k}\right\} \in Z_{\alpha}$, a contradiction. Thus condition (2.1) is also necessary. The proof of the theorem is now complete.

The Cauchy product of two series $\sum_{k=0}^{\infty} a_{k}, \sum_{k=0}^{\infty} b_{k}$ is $\sum_{k=0}^{\infty} c_{k}$ where

$$
c_{k}=a_{0} b_{k}+a_{1} b_{k-1}+\ldots+a_{k} b_{0}
$$

In the context of the sequence space $Z_{\alpha}$, we have the following theorem.

THEOREM 2.2. If $\left\{a_{k}\right\},\left\{b_{k}\right\} \in Z_{\alpha}$, so does their Cauchy product $\left\{c_{k}\right\}$

Proof. Consider the matrix

$$
A=\left[\begin{array}{cccccc}
a_{0} & 0 & 0 & 0 & . \\
a_{1} & a_{0} & 0 & 0 & . \\
a_{2} & a_{1} & a_{0} & 0 & \cdots \\
\ldots & \ldots & \ldots & \ldots & \ldots
\end{array}\right] .
$$

Noting that the $A$-transform of $\left\{b_{k}\right\}$ is $\left\{c_{k}\right\}$, since $\left\{a_{k}\right\} \in Z_{\alpha}$, $A \in\left(z_{\alpha}, z_{\alpha}\right)$ so that $\left\{c_{k}\right\} \in Z_{\alpha}$, since $\left\{b_{k}\right\} \in Z_{\alpha}$. 
Remark 2.1. (i) It is easy to establish Theorem 2.2, when $\alpha=1$, virtually by following the steps in the well-known Cauchy theorem on multiplication of series; Theorem 2.2 could be proved in the same way using (2.2).

(ii) Theorem 2.2 could be formally stated in the following form: If a sequence $\left\{a_{k}\right\}$ is given, then $\left\{c_{k}\right\} \in Z_{\alpha}$ for every sequence $\left\{b_{k}\right\} \in Z_{\alpha}$ if and only if $\left\{a_{k}\right\} \in z_{\alpha}$ where $c_{k}$ is defined by (2.6).

(iii) Theorem 2.2 is not true when $K=\mathbb{R}$ or $\psi^{\top}$ and $\alpha>1$ as illustrated by the following example. Let

$$
a_{k}=b_{k}=\frac{1}{(k+1)^{\frac{1}{2}\left(1+\frac{1}{\alpha}\right)}}, \alpha>1 \text {. }
$$

Then $\left\{a_{k}\right\},\left\{b_{k}\right\} \in Z_{\alpha}$ while $\left\{c_{k}\right\} \notin Z_{\alpha}$.

3. A Mercerian theorem.

We now set out to study the structure of $\left(z_{\alpha}, z_{\alpha}\right), \alpha \geq 1$, with a view to obtain a Mercerian theorem analogous to the one obtained earlier by Rangachari and Srinivasan [9]. $\left(z_{\alpha}, z_{\alpha}\right), \alpha \geq 1$, is a Banach algebra under the norm

$$
|| A||=\sup _{k \geq 0}\left(\sum_{n=0}^{\infty}\left|a_{n k}\right|^{\alpha}\right)^{\frac{1}{\alpha}}, A=\left(a_{n k}\right) \in\left(z_{\alpha}, z_{\alpha}\right),
$$

with the usual matrix addition, multiplication and elementwise scalar multiplication. First we note that if $A=\left(a_{n k}\right), B=\left(b_{n k}\right) \in\left(z_{\alpha}, z_{\alpha}\right)$, $(A B)_{n k}$ is defined, for, $(A B)_{n k}=\sum_{i=0}^{\infty} a_{n i} b_{i k}$ converges, since $b_{i k} \rightarrow 0$, $i \rightarrow \infty$ and $\sup _{n, i}\left|a_{n i}\right|<\infty$. We next show that $\left(z_{\alpha}, z_{\alpha}\right)$ is closed with respect to multiplication. For, using (2.2), 


$$
\begin{aligned}
\sum_{n=0}^{\infty}\left|(A B)_{n k}\right|^{\alpha} & =\sum_{n=0}^{\infty}\left|\sum_{i=0}^{\infty} a_{n i} b_{i k}\right|^{\alpha} \\
& \leq \sum_{n=0}^{\infty} \sum_{i=0}^{\infty}\left|a_{n i}\right|^{\alpha}\left|b_{i k}\right|^{\alpha} \\
& \left.=\sum_{i=0}^{\infty}\left|b_{i k}\right|^{\alpha} \sum_{n=0}^{\infty}\left|a_{n i}\right|^{\alpha}\right) \\
& \leq \|\left.\left. A\right|^{\alpha}|| B\right|^{\alpha}, k=0,1,2, \ldots .
\end{aligned}
$$

Thus $A B \in\left(z_{\alpha}, z_{\alpha}\right)$ and $\|A B\| \leq|| A|||| B||$. The associative law follows, for, if $A=\left(a_{n k}\right), B=\left(b_{n k}\right), c=\left(c_{n k}\right) \in\left(z_{\alpha}, z_{\alpha}\right)$,

$$
\begin{aligned}
\{(A B) C\}_{n k} & =\sum_{i=0}^{\infty}(A B)_{n i} c_{i k} \\
& =\sum_{i=0}^{\infty} c_{i k}\left(\sum_{j=0}^{\infty} a_{n j} b_{j i}\right) \\
& =\sum_{j=0}^{\infty} a_{n j}\left(\sum_{i=0}^{\infty} b_{j i} c_{i k}\right) \\
& =\sum_{j=0}^{\infty} a_{n j}(B C)_{j k} \\
& =\{A(B C)\}_{n k} .
\end{aligned}
$$

It remains to prove that $\left(z_{\alpha}, z_{\alpha}\right)$ is complete under the norm defined by (3.1). To see this, let $\left\{A^{(n)}\right\}$ be a Cauchy sequence in $\left(z_{\alpha}, z_{\alpha}\right)$ where $A^{(n)}=\left(a_{i j}(n)\right), i, j=0,1,2, \ldots$. Since $\left\{A^{n}\right\}$ is Cauchy, for any $\varepsilon>0$, there exists a positive integer $n_{0}$ such that for $m, n \geq n_{0}$,

$$
\| A^{(m)}-A^{(n)}||<\varepsilon \text {. }
$$

That is $\quad \sup _{j \geq 0} \sum_{i=0}^{\infty}\left|a_{i j}{ }^{(m)}-a_{i j}{ }^{(n)}\right|^{\alpha}<\varepsilon^{\alpha}$

Thus for all $i, j=0,1,2, \ldots$, 


$$
\left|a_{i j}{ }^{(m)}-a_{i j}^{(n)}\right|<\varepsilon, m, n \geq n_{o} .
$$

Hence $a_{i j}(n) \rightarrow a_{i j}, n \rightarrow \infty, i, j=0,1,2, \ldots$, since $K$ is complete. Consider the matrix $A=\left(\alpha_{i j}\right), i, j=0,1,2, \ldots$. For all $j=0,1,2, \ldots$,

$$
\sum_{i=0}^{k}\left|a_{i j}{ }^{(n)}\right|^{\alpha} \leq\left\|A^{(n)}\right\|^{\alpha} \leq M^{\alpha}, n, k=0,1,2, \ldots,
$$

where $M=\sup _{n \geq 0}|| A^{(n)} \|$. Allowing $n \rightarrow \infty$, we have,

$$
\sum_{i=0}^{k}\left|a_{i j}\right|^{\alpha} \leq M^{\alpha}, j, k=0,1,2, \ldots
$$

Allowing $k \rightarrow \infty$,

$$
\sum_{i=0}^{\infty}\left|a_{i j}\right|^{\alpha} \leq M^{\alpha}, j=0,1,2, \ldots,
$$

which shows that $A \in\left(l_{\alpha}, l_{\alpha}\right)$. Again for all $j, k=0,1,2, \ldots$,

$$
\sum_{i=0}^{k}\left|a_{i j}{ }^{(m)}-a_{i j}{ }^{(n)}\right|^{\alpha}<\varepsilon^{\alpha}, m, n \geq n_{0} .
$$

For $n \geq n_{0}$, allowing $m \rightarrow \infty$, for all $j, k=0,1,2, \ldots$,

$$
\sum_{i=0}^{k}\left|a_{i j}-a_{i j}(n)\right|^{\alpha} \leq \varepsilon^{\alpha} \text {. }
$$

Now, allowing $k \rightarrow \infty$, we have, for all $j=0,1,2, \ldots$,

$$
\sum_{i=0}^{\infty}\left|a_{i j}-a_{i j}{ }^{(n)}\right|^{\alpha} \leq \varepsilon^{\alpha}, n \geq n_{0} \text {. }
$$

That is, $\quad \sup _{j \geq 0}\left(\sum_{i=0}^{\infty}\left|a_{i j}-a_{i j}{ }^{(n)}\right|^{\alpha}\right)^{1 / \alpha} \leq \varepsilon, n \geq n_{0}$.

That is, $\quad\left\|A^{(n)}-A\right\| \leq \varepsilon, \quad n \geq n_{0}$,

which shows that $A^{(n)} \rightarrow A, n \rightarrow \infty$.

The Mercerian theorem mentioned is the following.

THEOREM 3.1. When $K=Q_{p}$, the p-adic field for a prime $p$, if $y_{n}=x_{n}+\lambda p^{n}\left(x_{0}+x_{1}+\ldots+x_{n}\right)$ and $\left\{y_{n}\right\} \in Z_{\alpha}$, then $\left\{x_{n}\right\} \in Z_{\alpha}$ if 
$|\lambda|_{p}<\left(1-\rho^{\alpha}\right)^{1 / \alpha}$ where $\rho=|p|_{p}<1$.

Proof. Since $\left(z_{\alpha}, z_{\alpha}\right)$ is a Banach algebra, if $\lambda \in Q_{p}$ is such that $|\lambda|_{p}<\frac{1}{\|A\|}, A \in\left(\tau_{\alpha}, z_{\alpha}\right)$, then $I-\lambda A$, where $I$ is the identity matrix, has an inverse in $\left(z_{\alpha}, z_{\alpha}\right)$. The matrix of transformation is $I+\lambda A$ where

$$
A \equiv\left[\begin{array}{cccccc}
1 & 0 & 0 & 0 & . & . \\
p & p & 0 & 0 & . & . \\
p^{2} & p^{2} & p^{2} & 0 & \ldots \\
\ldots & \ldots & \ldots & \ldots & . & .
\end{array}\right] .
$$

We note that $A \in\left(I_{\alpha}, Z_{\alpha}\right)$ with $\|A\|=\frac{1}{\left(1-\rho^{\alpha}\right)^{1 / \alpha}}$. Then $I+\lambda A$ has an inverse in $\left(z_{\alpha}, z_{\alpha}\right)$ if $|\lambda|_{p}<\left(1-\rho^{\alpha}\right)^{1 / \alpha}$. Since $y=(I+\lambda A) x$ where $y=\left\{y_{k}\right\}, x=\left\{x_{k}\right\}$ and lower triangular matrices are associative, it follows that $(I+\lambda A)^{-1} y=x$. Since $y \in z_{\alpha}$ and $(I+\lambda A)^{-1} \in\left(Z_{\alpha}, Z_{\alpha}\right)$, it follows that $x \in Z_{\alpha}$.

The proof of the theorem is now complete.

4. A Steinhaus type theorem for $\tau_{\alpha}$.

Theorem 4.2 to follow is a steinhaus type result proved using the characterization of $\left(z_{\alpha}, z_{\alpha}\right)$ in Theorem 2.1 . We write $A \in\left(l_{\alpha}, z_{\alpha} ; P\right)$ if $A \in\left(z_{\alpha}, z_{\alpha}\right)$ and $\sum_{n=0}^{\infty}(A x)_{n}=\sum_{k=0}^{\infty} x_{k}, x=\left\{x_{k}\right\} \in z_{\alpha} ; A \in\left(z_{\alpha}, z_{\alpha} ; P\right)^{\prime}$ if $A \in\left(Z_{\alpha}, z_{\alpha} ; P\right)$ and $a_{n k} \rightarrow 0, k \rightarrow \infty, n=0,1,2, \ldots$. It is easy to check that $A \in\left(z_{\alpha}, z_{\alpha} ; P\right)$ if and only if $A \in\left(z_{\alpha}, z_{\alpha}\right)$ and $\sum_{n=0}^{\infty} a_{n k}=1$, $k=0,1,2, \ldots$.

THEOREM 4.1. If $A \in\left(Z_{\alpha}, z_{\alpha}\right)$ such that $a_{n k} \rightarrow 0, k \rightarrow \infty$, 
$n=0,1,2, \ldots$ and $\overline{\lim }_{k \rightarrow \infty} \sum_{n=0}^{\infty}\left|a_{n k}\right|^{\alpha}>0$, then there exists a sequence $x=\left\{x_{k}\right\} \in \tau_{\beta}, \beta>\alpha, A x=\left\{(A x)_{n}\right\} \& \tau_{\alpha}$.

Proof. By hypothesis, for some $\varepsilon>0$, there exists a subsequence $\{k(i)\}$ of positive integers such that

$$
\sum_{n=0}^{\infty}\left|a_{n, k(i)}\right|^{\alpha} \geq 2 \varepsilon, i=1,2, \ldots
$$

In particular,

$$
\sum_{n=0}^{\infty}\left|a_{n, k(1)}\right|^{\alpha} \geq 2 \varepsilon
$$

Choose a positive integer $n(1)$ such that

$$
\sum_{n=n(1)+1}^{\infty}\left|a_{n, k(1)}\right|^{\alpha}<\min \left(2^{-1}, \frac{\varepsilon}{2}\right),
$$

so that

$$
\sum_{n=0}^{n(1)}\left|a_{n, k(1)}\right|^{\alpha}>\varepsilon
$$

More generally, having chosen the positive integers $k(j), n(j), j \leq m-1$, choose a positive integer $k(m)$ such that $k(m)>k(m-1)$,

$$
\begin{aligned}
& \sum_{n=0}^{\infty}\left|a_{n, k(m)}\right|^{\alpha} \geq 2 \varepsilon, \\
& \sum_{n=0}^{n(m-1)}\left|a_{n, k(m)}\right|^{\alpha}<\min \left(2^{-m}, \frac{\varepsilon}{2}\right),
\end{aligned}
$$

and then choose a positive integer $n(m)$ such that $n(m)>n(m-1)$,

$$
\sum_{n=n(m)+1}^{\infty}\left|a_{n, k(m)}\right|^{\alpha}<\min \left(2^{-m}, \frac{\varepsilon}{2}\right),
$$

so that

$$
\sum_{n=n(m-1)+1}^{n(m)}\left|a_{n, k(m)}\right|^{\alpha}>2 \varepsilon-\frac{\varepsilon}{2}-\frac{\varepsilon}{2}=\varepsilon .
$$

Since $K$ is non-trivially valued, there exists $\pi \in K$ such that $0<\rho=|\pi|<1$. For each $i=1,2, \ldots$, choose a non-negative integer $\lambda(i)$ such that 


$$
\rho^{\lambda(i)+1} \leq \frac{1}{i^{1 / \alpha}}<\rho^{\lambda(i)} .
$$

If the sequence $x=\left\{x_{k}\right\}$ is defined by

$$
\begin{aligned}
x_{k}=\pi^{\lambda(i)}, & k=k(i) \\
& =0, k \neq k(i)
\end{aligned} \mid, \quad i=1,2, \ldots,
$$

$\left\{x_{k}\right\} \in Z_{\beta} \backslash z_{\alpha}$, for,

$$
\begin{aligned}
\sum_{k=0}^{\infty}\left|x_{k}\right|^{\beta}=\sum_{i=1}^{\infty}\left|x_{k(i)}\right|^{\beta} & =\sum_{i=1}^{\infty} \rho^{\beta \lambda(i)} \\
& \leq \frac{1}{\rho^{\beta}} \sum_{i=1}^{\infty} \frac{1}{i^{\beta / \alpha}}<\infty,
\end{aligned}
$$

since $\beta>\alpha$, while,

$$
\sum_{k=0}^{\infty}\left|x_{k}\right|^{\alpha}=\sum_{i=1}^{\infty}\left|x_{k(i)}\right|^{\alpha}=\sum_{i=1}^{\infty} \rho^{\alpha \lambda(i)}>\sum_{i=1}^{\infty} \frac{1}{i}=\infty .
$$

Defining $n_{0}=0$,

$$
\begin{aligned}
& \sum_{n=0}^{n(N)}\left|(A x)_{n}\right|^{\alpha} \geq \sum_{m=1}^{N} \underset{n=n(m-1)+1}{n(m)}\left|\sum_{i=1}^{\infty} a_{n, k(i)} x_{k(i)}\right|^{\alpha} \\
& =\left.\sum_{m=1}^{N} \underset{n=n(m-1)+1}{n(m)}\right|_{i=1} ^{\infty} a_{n,\left.k(i) \pi^{\lambda(i)}\right|^{\alpha}} \\
& \geq \sum_{m=1}^{N} \sum_{n=n(m-1)+1}^{n(m)}\left\{\left|a_{n, k(m)}\right|^{\alpha} \rho^{\alpha \lambda(m)}\right. \\
& \left.-\sum_{i \neq m}\left|a_{n, k(i)}\right|^{\alpha}{ }^{\alpha \lambda(i)}\right\} \\
& \text { (using (2.2)) } \\
& \geq \sum_{m=1}^{N} \underset{n=n(m-1)+1}{n(m)}\left\{\left|a_{n, k(m)}\right|^{\alpha} m^{-1}\right. \\
& \left.-\frac{1}{\rho} \sum_{i \neq m}\left|a_{n, k(i)}\right|^{\alpha}\right\} \\
& >\sum_{m=1}^{N} \epsilon m^{-1}-\frac{1}{\rho^{\alpha}} \sum_{m=1}^{N} \sum_{n=n(m-1)+1}^{n(m)} \sum_{i \neq m}\left|a_{n, k(i)}\right|^{\alpha} .
\end{aligned}
$$


We note that

$$
\begin{aligned}
\sum_{m=1}^{\infty} \sum_{n=n(m-1)+1}^{n(m)} \sum_{i<m}\left|a_{n, k(i)}\right|^{\alpha} & =\sum_{m=1}^{\infty} \sum_{n=n(m)+1}^{\infty}\left|a_{n, k(m)}\right|^{\alpha} \\
& \leq \sum_{m=1}^{\infty} 2^{-m} .
\end{aligned}
$$

Similarly it can be shown that

$$
\sum_{m=1}^{\infty} \underset{n=n(m-1)+1}{n} \sum_{i>m}^{n(m)}\left|a_{n, k(i)}\right|^{\alpha}<\sum_{m=1}^{\infty} 2^{-(m+1)} .
$$

Thus it follows from (4.1) to (4.3) that

$$
\sum_{n=0}^{n(N)}\left|(A x)_{n}\right|^{\alpha}>c \sum_{m=1}^{N} \frac{1}{m}-\frac{3}{2}
$$

Since $\sum_{m=1}^{\infty} \frac{1}{m}=\infty$, it follows that $\left\{(A x)_{n}\right\} \notin \tau_{\alpha}$.

THEOREM 4.2. $\left(z_{\alpha}, z_{\alpha} ; P\right)^{\prime} \cap\left(z_{\beta}, z_{\alpha}\right)=\varnothing, \beta>\alpha$.

Proof. Suppose $A \in\left(z_{\alpha}, z_{\alpha} ; P\right) ' \cap\left(z_{\beta}, z_{\alpha}\right)^{\prime}, \beta>\alpha$. Then

$\sum_{n=0}^{\infty}\left|a_{n k}\right|^{\alpha} \geq\left|\sum_{n=0}^{\infty} a_{n k}\right|^{\alpha}=1, k=0,1,2, \ldots$ so that $\overline{1 i m}_{k \rightarrow \infty}^{\infty} \sum_{n=0}^{\infty}\left|a_{n k}\right|^{\alpha} \geq 1$.

By Theorem 4.1, there exists $x=\left\{x_{k}\right\} \in Z_{\beta}$ such that $\left\{(A x)_{n}\right\} \& Z_{\alpha}$,

a contradiction.

Remark 4.1. When $K=\mathbb{R}$ or $\ell$, it was proved by Fridy [3] that $\left(z_{1}, z_{1} ; P\right) \cap\left(z_{\alpha}, z_{1}\right)=\emptyset, \alpha>1$. This result, as such, Eails to hold when $K$ is a complete, non-trivially valued, non-archimedean field, as the following example shows. Let $K=Q_{3}$ and $A=\left(a_{n k}\right)$ where

$$
a_{n k}=\frac{1}{4}\left(\frac{3}{4}\right)^{n}, n, k=0,1,2, \ldots \text {. }
$$

$\sup _{k \geq 0} \sum_{n=0}^{\infty}\left|a_{n k}\right|_{3}=\frac{1}{1-\rho}<\infty$ where $\rho=|3|_{3}$ and $\sum_{n=0}^{\infty} a_{n k}=1, k=0,1,2, \ldots$, so that $A \in\left(Z_{1}, Z_{1} ; P\right)$; but, for $\alpha>1$, if $x=\left\{x_{k}\right\} \in Z_{\alpha}$, 


$$
\begin{aligned}
\sum_{n=0}^{\infty}\left|(A x)_{n}\right|_{3} & =\sum_{n=0}^{\infty}\left|\sum_{k=0}^{\infty} a_{n k} x_{k}\right|_{3} \\
& =\sum_{n=0}^{\infty}\left|\frac{1}{4}\left(\frac{3}{4}\right)^{n}\right|_{3}\left|\sum_{k=0}^{\infty} x_{k}\right|_{3} \\
& =\left|\sum_{k=0}^{\infty} x_{k}\right|_{3} \frac{1}{1-0}<\infty \text { (since }\left\{x_{k}\right\} \in l_{\alpha},
\end{aligned}
$$

$x_{k} \rightarrow 0, k \rightarrow \infty$ and so $\sum_{k=0}^{\infty} x_{k}$ converges as $K=Q_{3}$ is complete).

Thus $A \in\left(z_{\alpha}, z_{1}\right)$ also.

Remark 4.2. $\left(z_{\alpha}, z_{\alpha} ; P\right) \cap\left(l_{\infty}, z_{\alpha}\right)=\emptyset$ where $l_{\infty}$ is the space of all bounded sequences with entries in $K$. For, if $A \in\left(l_{\alpha}, z_{\alpha} ; P\right) \cap\left(l_{\infty}, l_{\alpha}\right)$, then $A \in\left(z_{\alpha}, z_{\alpha} ; p\right)$ ' $\cap\left(z_{\beta}, z_{\alpha}\right), \beta>\alpha$, a contradiction.

Remark 4.3. Virtually by following the proof of Theorem 4.1, we can show that given any matrix $A \in\left(l_{\alpha}, l_{\alpha} ; P\right)$, there exists a sequence of $O^{\prime} s$ and $I$ 's whose $A$-transform is not in $\tau_{\alpha}$. This is analogous to Schur's version of the well-known steinhaus theorem for regular matxices (see [10], when $K=\mathbb{R}$ or $\ell$ and [8] when $K$ is a complete, nontrivially valued, non-archimedean field).

\section{References}

[1] G. Bachman, Introduction to p-adic numbers and valuation theory, Academic Press, 1964.

[2] J. A. Fridy, "A note on absolute summability", Proc. Amer. Math. Soc. 20 (1969), 285-286.

[3] J. A. Fridy, "Properties of absolute summability matrices", Proc. Amer. Math. Soc. 24 (1970), 583-585.

[4] K. Knopp, G. G. Lorentz, "Beiträge zur absoluten Limitierung", Arch. Math. 2 (1949), 10-16.

[5] M. Koskela, "A characterization of non-negative matrix operators on $\imath^{p}$ to $l^{q}$ with $\infty>p \geq q>1^{\prime \prime}$, Pacific J. Math. 75 (1978), 165-169. 
[6] I. J. Moddox, EZements of Functional Analysis, Cambridge, 1977.

[7] F. M. Mears, "Absolute regularity and the Nörlund mean", Ann. of Math. 38 (1937), 594-601.

[8] P. N. Natarajan, "The Steinhaus theorem for Toeplitz matrices in non-archimedean fields", Comment. Math. Prace Mat. 20 (1978), 417-422.

[9] M. S. Rangachari, V. K. Srinivasan, "Matrix transformations in non-archimedean fields", Indag. Math. 26 (1964), 422-429.

[10] I. Schur, "Über lineare Transformationen in der Theorie der unendlichen Reihen", J. Reine Angew. Math. 151 (1921), 79-111.

[11] M. Steiglitz, H. Tietz, "Matrix transformationen von Folgenräumen eine Ergebnisübersicht", Math. Z. 154 (1977), 1-16.

Department of Mathematics,

Vivekananda College,

Madras - $600 \quad 004$

India. 